\title{
Compact Einstein-Weyl four-dimensional manifolds
}

\author{
Guy Bonneau*
}

June 25, 2018

\begin{abstract}
We look for four dimensional Einstein-Weyl spaces equipped with a regular Bianchi metric. Using the explicit 4-parameters expression of the distance obtained in a previous work for non-conformally-Einstein Einstein-Weyl structures, we show that only four 1-parameter families of regular metrics exist on orientable manifolds : they are all of Bianchi $I X$ type and conformally Kähler ; moreover, in agreement with general results, they have a positive definite conformal scalar curvature. In a Gauduchon's gauge, they are compact and we obtain their topological invariants. Finally, we compare our results to the general analyses of Madsen, Pedersen, Poon and Swann : our simpler parametrisation allows us to correct some of their assertions.
\end{abstract}

PACS : 02.40.Hw, 04.20.Jb

Keywords : Einstein-Weyl, Bianchi, conformal, Káhler, compact. 


\section{Introduction}

In the last years, Einstein-Weyl geometry has deserved some interest. A Weyl space [1] is a conformal manifold with a torsion-free connection $\mathrm{D}$ and a one-form $\gamma$ such that for each representative metric $\mathrm{g}$ in a conformal class $[\mathrm{g}]$,

$$
D_{\mu} g_{\nu \rho}=\gamma_{\mu} g_{\nu \rho}
$$

A different choice of representative metric : $g \longrightarrow \tilde{g}=e^{f} g$ is accompanied by a change in $\gamma: \gamma \longrightarrow \tilde{\gamma}=\gamma+d f$. Conversely, if the one-form $\gamma$ is exact, the metric $\mathrm{g}$ is conformally equivalent to a Riemannian metric $\tilde{g}: D_{\mu} \tilde{g}_{\nu \rho}=0$. In that case, we shall speak of an exact Weyl structure. Einstein-Weyl spaces are defined by :

$$
\begin{aligned}
\mathcal{R}_{(\mu \nu)}^{(D)} & =\Lambda^{\prime} g_{\mu \nu} \Leftrightarrow \\
R_{\mu \nu}^{(\nabla)}+\nabla_{(\mu} \gamma_{\nu}+\frac{1}{2} \gamma_{\mu} \gamma_{\nu} & =\Lambda g_{\mu \nu} \quad, \quad \Lambda=\Lambda^{\prime}-\frac{1}{2}\left[\nabla_{\lambda} \gamma^{\lambda}-\gamma_{\lambda} \gamma^{\lambda}\right] .
\end{aligned}
$$

This condition is a (conformally invariant) generalisation of the usual Einstein condition. Note that for an exact Einstein-Weyl structure, $\gamma=d f$, the representative metric is conformally Einstein (for recent reviews see refs. 1, 2, 3, 4). Let us also recall that, in the compact case, on general grounds, strong results have been known for some time :

- There exits a unique metric in a given conformal class [g] such that the Weyl form is co-closed [5],

$$
\nabla_{\mu} \gamma^{\mu}=0
$$

One then speaks of the "Gauduchon's gauge" and of a "Gauduchon's metric".

- The analysis of Einstein-Weyl equations in this gauge gives two essential results :

- The dual of the Weyl form $\gamma$ is a Killing vector [6]: $\nabla_{(\mu} \gamma_{\nu)}=0$,

- Four dimensional Einstein-Weyl space have a constant conformal scalar curvature [7, 8, 9].

On the other hand, in a recent work [10, we studied in a purely local approach, (non-)compact 4-dimensional Einstein-Weyl structures on cohomogenity-one manifolds with a 3 dimensional group of isometries transitive on codimension-one surfaces, i.e., in the general relativity terminology, Bianchi metrics [11, 12], and neither require compactness nor a diagonal form for the metric. We however got interesting results and proved that for all class A Bianchi metrics, there exits a simple Gauduchon's gauge such that the conformal scalar curvature is constant on the manifold. Our results are summarised in a :

Theorem : (Non-)compact Einstein-Weyl Bianchi metrics of the types $V I_{0}, V I I_{0}, V I I I$ and $I X$ are conformally Kähler or conformally Einstein. In the non-exact Einstein-Weyl case, the metric may be taken in a diagonal form and is given in equ. (3). The conformal scalar curvature has a constant sign on the manifold and, in our particular Gauduchon's gauge, the dual of the Weyl form is a Killing vector.

$$
d s^{2}=\frac{2}{\Gamma}\left[\frac{k-n^{33} x}{\Omega^{2}(x)\left[1+x^{2}\right]^{2}}(d x)^{2}+\frac{k-n^{33} x}{\left[1+x^{2}\right]}\left[\left(\sigma^{1}\right)^{2}+\left(\sigma^{2}\right)^{2}\right]+\frac{\Omega^{2}(x)}{k-n^{33} x}\left(\sigma^{3}\right)^{2}\right],
$$




$$
\begin{aligned}
\gamma & = \pm \frac{2 \Omega^{2}(x)}{k-n^{33} x} \sigma^{3} ; d \sigma^{i}=\frac{1}{2} n^{i i} \epsilon_{i j k} \sigma^{j} \wedge \sigma^{k} \quad, \quad i, j, k=1,2,3 \\
n^{11} & =n^{22}=1, n^{33}=0\left(\text { Bianchi } V I I_{0}\right),-1(V I I I),+1(I X) ; \\
\Omega^{2}(x) & =n^{33}+\left[n^{33}\left(x^{2}-1\right)-2 k x\right]\left[l_{1}+l_{2} \arctan (x)\right]+l_{2}\left[n^{33} x-2 k\right] .
\end{aligned}
$$

Let us recall from [10] that $\arctan (x)$ belongs to $(-\pi / 2,+\pi / 2)$, thanks to the integration constant $l_{1}$; moreover, $l_{1}, l_{2}, k$ and the homothetic positive parameter $\Gamma$ are arbitrary constants, and, in our particular Gauduchon's gauge, the conformal scalar curvature is the constant

$$
S^{D}=4 \Gamma l_{2}
$$

It is then natural to look for regular, complete metrics among our solutions. The further requirement of compactness would a priori be a gauge dependent condition : as we shall see, all complete metrics in our Gauduchon's gauge are in fact compact ones. This confirms that our choice does correspond to the unique Gauduchon's gauge of [5].

In this work, using the terminology of Gibbons and Hawking [13] on nuts and bolts, well adapted to the analysis of the completeness of our candidate metrics on orientable manifolds, we prove that in the Bianchi class A, up to an arbitrary homothetic factor $\Gamma>0$, there exist four, and only four, one-parameter families of complete Einstein-Weyl metrics with a non-exact Weyl form. More precisely, given a positive conformal scalar curvature $S^{D} \equiv 4 \Gamma l_{2}$ 円, the parameters in equation (3) are fixed and in particular the metrics are Bianchi $I X$ ones. The absence of any complete metrics in Bianchi $V I I_{0}$ and $V I I I$ subclasses agrees with refs. [3, 4] that show that the compactness of the metric requires a compact symmetry group. The four families are respectively nut-nut, nut-bolt, bolt-nut or bolt-bolt.

The paper is organised as follows : in the next Section, we study the regularity for Bianchi $V I I I$ or $I X$ metrics and discuss their completeness. For Bianchi $V I I_{0}$ candidates, we show in Section 3 that they cannot be regular and finally, after a comparison with previous results 9, 3, 4, conclude with some comments on the topological properties of our compact solutions and the underlying manifolds in Section 4.

\section{Bianchi $V I I I$ and $I X$ complete metrics?}

Let us introduce the new "proper time"

$$
t=k-n^{33} x
$$

The distance becomes :

$$
\begin{aligned}
d s^{2} & =\frac{2}{\Gamma}\left[\frac{t}{\tilde{\Omega}^{2}(t)\left[1+(t-k)^{2}\right]^{2}}(d t)^{2}+\frac{t}{\left[1+(t-k)^{2}\right]}\left[\left(\sigma^{1}\right)^{2}+\left(\sigma^{2}\right)^{2}\right]+\frac{\tilde{\Omega}^{2}(t)}{t}\left(\sigma^{3}\right)^{2}\right], \\
\tilde{\Omega}^{2}(t) & =n^{33}+\left(n^{33} l_{1}-l_{2} \arctan (t-k)\right)\left[t^{2}-\left(1+k^{2}\right)\right]-l_{2}(t+k)
\end{aligned}
$$

In our local approach, the "time" interval results from the positivity of the distance (6) : this leads to the two conditions

$$
t>0, \tilde{\Omega}^{2}(t)>0
$$

\footnotetext{
1 Looking for complete metrics, we explicitely checked a theorem given by Pedersen and Swann [9, 6, 5], asserting that a compact non-exact four-dimensional Einstein-Weyl space should have a strictly positive conformal scalar curvature.
} 
As

$$
\frac{d \tilde{\Omega}^{2}(t)}{d t}=2 t g(t), \quad g(t)=n^{33} l_{1}-l_{2}\left[\arctan (t-k)+\frac{t-k}{1+(t-k)^{2}}\right], \frac{d g(t)}{d t}=\frac{-2 l_{2}}{\left[1+(t-k)^{2}\right]^{2}}
$$

the discussion separates into three subcases according to the behaviour of $\tilde{\Omega}^{2}(t)$ when $t$ varies. Let

$$
\delta=g(+\infty)=n^{33} l_{1}-\frac{\pi}{2} l_{2} \text { and } \mu=g(0)=n^{33} l_{1}+l_{2}\left(\arctan k+\frac{k}{1+k^{2}}\right):
$$

- a) $\delta \geq 0$ and $\mu \geq 0$ : $\tilde{\Omega}^{2}(t)$ monotonicaly increases with $t$.

- b) $\delta \leq 0$ and $\mu \leq 0$ : $\tilde{\Omega}^{2}(t)$ monotonicaly decreases with $t$.

- c) $\delta \mu<0: \tilde{\Omega}^{2}(t)$ has a single extremum when $t$ varies (obtained when $\mathrm{g}(\mathrm{t})$ vanishes). The extremum is a maximum if $l_{2}$ and $\mu>0$ (case $c^{+}$) and a minimum if $l_{2}$ and $\mu<0$ (case $\left.c^{-}\right)$.

The behaviour of the distance at $\infty$ is readily seen to be singular if $\delta \neq 0$. Indeed,

$$
d s^{2} \sim \frac{2}{\Gamma}\left[\frac{(d t)^{2}}{\delta t^{5}}+\frac{1}{t}\left[\left(\sigma^{1}\right)^{2}+\left(\sigma^{2}\right)^{2}\right]+\delta t\left(\sigma^{3}\right)^{2}\right], \quad t \rightarrow+\infty
$$

and the change of variable $\rho=(t)^{-3 / 2}$ leaves a non removable singularity at $\rho=0$.

Consider now the special case when $\delta$ vanishes : the function $\tilde{\Omega}^{2}(t)$ goes to $n^{33}$ when $t \rightarrow+\infty$. So, $n^{33}$ should be +1 , and the distance behaves as

$$
d s^{2} \sim \frac{2}{\Gamma}\left[\frac{(d t)^{2}}{t^{3}}+\frac{1}{t}\left[\left(\sigma^{1}\right)^{2}+\left(\sigma^{2}\right)^{2}+\left(\sigma^{3}\right)^{2}\right] \quad t \rightarrow+\infty\right.
$$

which gives a nut 13] after the change $\rho=t^{-1 / 2}$ :

$$
d s^{2} \sim \frac{8}{\Gamma}\left[(d \rho)^{2}+\frac{\rho^{2}}{4}\left[\left(\sigma^{1}\right)^{2}+\left(\sigma^{2}\right)^{2}+\left(\sigma^{3}\right)^{2}\right]\right] \quad, \rho \rightarrow 0
$$

The singularity is removable if one choses cartesian coordinates rather than polar ones. Note that in other gauges, the metric is still regular, but, for example in the "Kähler gauge", a boundary appears at infinity as the metric is asymptotically euclidean:

$d \tilde{s}^{2}=\frac{\Gamma}{2}\left[1+(t-k)^{2}\right] d s^{2} \sim \frac{(d t)^{2}}{t}+t\left[\left(\sigma^{1}\right)^{2}+\left(\sigma^{2}\right)^{2}+\left(\sigma^{3}\right)^{2}\right]=4(d \sqrt{t})^{2}+(\sqrt{t})^{2}\left[\left(\sigma^{1}\right)^{2}+\left(\sigma^{2}\right)^{2}+\left(\sigma^{3}\right)^{2}\right], t \rightarrow \infty$.

To sum up, we have

Lemma 1 : if the proper time interval extends to $+\infty$, the metric can be regular only in the Bianchi $I X$ case with $l_{1}=\frac{\pi}{2} l_{2}$.

Consider now the behaviour of the distance near $t=0$ supposed to be the lowest possible value of the proper time compatible with a positive metric. If $\tilde{\Omega}^{2}(0)>0$, the distance write - after the change of variable $\rho=(t)^{3 / 2}$ :

$$
d s^{2} \sim \frac{2}{\Gamma}\left[\frac{4(d \rho)^{2}}{9 \tilde{\Omega}^{2}(0)\left[1+k^{2}\right]^{2}}+\frac{\rho^{2 / 3}}{\left[1+k^{2}\right]}\left[\left(\sigma^{1}\right)^{2}+\left(\sigma^{2}\right)^{2}\right]+\frac{\tilde{\Omega}^{2}(0)}{\rho^{2 / 3}}\left(\sigma^{3}\right)^{2}\right], \rho \rightarrow 0
$$


and is singular. Then we are left with the case $\tilde{\Omega}^{2}(0)=0$, where one finds :

$$
\frac{d \tilde{\Omega}^{2}}{d t}(0)=0, \quad \frac{d^{2} \tilde{\Omega}^{2}}{d t^{2}}(0)=\frac{2 n^{33}}{1+k^{2}}
$$

and here again the positivity requires $n^{33}=+1$. Then one can change the variable $t$ into $\rho$ given by

$$
\rho=\sqrt{t}
$$

and get the following nut behaviour for the distance near $t=0$ :

$$
d s^{2} \sim \frac{8}{\Gamma\left[1+k^{2}\right]}\left[(d \rho)^{2}+\frac{\rho^{2}}{4}\left[\left(\sigma^{1}\right)^{2}+\left(\sigma^{2}\right)^{2}+\left(\sigma^{3}\right)^{2}\right]\right], \rho \rightarrow 0 .
$$

At last, singularities in the distance may occur at zeroes of $\Omega^{2}(x)$ and, the range of variation of the proper time being limited by such zeroes, one has to discuss the nature of the singularity at these values, still using the work of Gibbons and Hawking on nuts and bolts 13 to check the completeness of the metric at both ends.

If $\tilde{\Omega}^{2}(\alpha)=0$ with $\frac{d \tilde{\Omega}^{2}}{d t}(\alpha)=0$ and $\alpha \neq 0$, positivity enforces $\alpha$ to be a minimum ; then, $\tilde{\Omega}^{2}(0)>0$ which, as explained above, gives a singularity. So, consider the situation with $\frac{d \tilde{\Omega}^{2}}{d t}(\alpha) \neq 0$ and change the variable $t$ to $\rho$ according to :

$$
t=\alpha+\rho^{2} \frac{d \tilde{\Omega}^{2}}{d t}(\alpha)
$$

using $\tilde{\Omega}^{2}(t) \sim \rho^{2}\left[\frac{d \tilde{\Omega}^{2}}{d t}(\alpha)\right]^{2}$, the distance behaves when $\rho \rightarrow 0$ as :

$$
\begin{aligned}
d s^{2} \sim \frac{8 \alpha}{\Gamma\left[1+(\alpha-k)^{2}\right]^{2}}\left[(d \rho)^{2}\right. & +\frac{\rho^{2}}{4}\left(\frac{1+(\alpha-k)^{2}}{\alpha} \frac{d \tilde{\Omega}^{2}}{d t}(\alpha)\right)^{2}\left(\sigma^{3}\right)^{2}+ \\
& \left.+\frac{1+(\alpha-k)^{2}}{4}\left[\left(\sigma^{1}\right)^{2}+\left(\sigma^{2}\right)^{2}\right]\right]
\end{aligned}
$$

and one has a bolt of twist $p$ iff. :

$$
\frac{1+(\alpha-k)^{2}}{\alpha} \frac{d \tilde{\Omega}^{2}}{d t}(\alpha)= \pm p, \quad p=1,2 \ldots
$$

Indeed, in such a case, restricting the range in the angle $\psi$ coresponding to $\sigma^{3}=d \psi+\cos \theta d \phi$, e.t.c... to the interval $[0,4 \pi / p]$, there is no longer a polar singularity in the distance. This condition corresponds to :

$$
g(\alpha)= \pm \frac{p}{2\left[1+(\alpha-k)^{2}\right]} \quad, \quad p=1,2, \ldots
$$

We have now all the building blocks needed in our discussion on the regularity of our EinsteinWeyl metrics. 
First, the absence of a non removable singularity at $t=0$ supposes that :

$$
\tilde{\Omega}^{2}(0) \leq 0 \Leftrightarrow n^{33} l_{1}+l_{2}\left(\arctan k+\frac{k}{1+k^{2}}\right) \geq \frac{n^{33}}{1+k^{2}}
$$

We now study successively the cases a), b), $c^{+}$) and $c^{-}$).

- case a) : $\tilde{\Omega}^{2}(t)$ monotonicaly increasing :

the range of $t$ is $\left[\alpha,+\infty\left[; \alpha \geq 0, \tilde{\Omega}^{2}(\alpha)=0\right.\right.$.

Lemma 1 enforces : $n^{33}=+1, l_{1}=\frac{\pi}{2} l_{2}$; then we have a nut at $\infty$. Moreover, thanks to (13), the relation :

$$
l_{1}+l_{2}\left(\arctan k+\frac{k}{1+k^{2}}\right) \equiv l_{2}\left(\frac{\pi}{2}+\arctan k+\frac{k}{1+k^{2}}\right) \geq \frac{1}{1+k^{2}}
$$

implies $l_{2}>0$, in agreement with the aforementioned theorem [9].

$-\boldsymbol{\alpha}=\mathbf{0}$ : we have a nut-nut family of metrics (depending on a positive parameter $l_{2}$ ), whose distance is given by the expression (3) with :

$$
n^{33}=+1 \quad, \quad l_{1}=\frac{\pi}{2} l_{2} ;
$$

$k$ given by the unique solution of $\quad: \frac{\pi}{2}+\arctan k+\frac{l_{2} k-1}{l_{2}\left(1+k^{2}\right)}=0$.

In particular $k>-l_{2}$.

$-\boldsymbol{\alpha}>0$ : a complete metric needs $\alpha$ being a nut or a bolt of twist 1 . Here, as $\alpha>0, \frac{d \tilde{\Omega}^{2}}{d t}(\alpha)>0$ and only a bolt is possible : we have a bolt-nut family of metrics (depending on a positive parameter $l_{2}$ ). The bolt condition (12) and the vanishing of $\widetilde{\Omega}^{2}(\alpha)$ uniquely determine the values of $\alpha$ and $k$ and we have the following values for the parameters in the distance (3) :

$$
\begin{aligned}
& n^{33}=+1, l_{1}=\frac{\pi}{2} l_{2}, \quad k=\frac{1+4 l_{2} x_{1}+3 x_{1}^{2}}{2\left(2 l_{2}+x_{1}\right)}, \quad \alpha=k-x_{1}, \\
& x_{1}>-2 l_{2} \text { is the unique solution of }: \frac{\pi}{2}+\arctan x_{1}+\frac{2 l_{2} x_{1}-1}{2 l_{2}\left(1+x_{1}^{2}\right)}=0,
\end{aligned}
$$

In particular, the position of the bolt is given by

$$
\alpha=\frac{1+x_{1}^{2}}{2\left(2 l_{2}+x_{1}\right)} \geq \frac{1}{2 l_{2}+\sqrt{4 l_{2}^{2}+1}} .
$$

- case b) : $\tilde{\Omega}^{2}(t)$ monotonicaly decreasing :

the distance cannot be positive and this situation is excluded.

- case $\left.c^{+}\right)$: $\tilde{\Omega}^{2}(t)$ increases to a maximum value reached for $t=\beta, g(\beta)=$ $0, \tilde{\Omega}^{2}(\beta)>0$ then decreases and vanishes at $\alpha^{\prime}, \tilde{\Omega}^{2}\left(\alpha^{\prime}\right)=0$; the range of $t$ is $\left[\alpha, \alpha^{\prime}\right]$. Moreover, a positive maximum requires $l_{2}$ and $n^{33}>0$, i.e. $n^{33}=+1$. 
$-\boldsymbol{\alpha}=\mathbf{0}$ : we have a nut-bolt of twist 1 (replace $\pm p$ in equ.(12) by - 1) family of metrics (depending on a positive parameter $l_{2}$ ). The previous discussion enforces :

$$
n^{33}=+1, \mu=n^{33} l_{1}+l_{2}\left(\arctan k+\frac{k}{1+k^{2}}\right)=\frac{1}{1+k^{2}}, \delta=l_{1}-\frac{\pi}{2} l_{2}<0 .
$$

The existence of $k$ then requires $l_{1}+\frac{\pi}{2} l_{2}>0$. Finally, adding the bolt condition (12) and the vanishing of $\tilde{\Omega}^{2}\left(\alpha^{\prime}\right), \alpha^{\prime}$ and $k$ are uniquely $f$ determined and we have the following values for the parameters in the distance (3):

$$
\begin{aligned}
n^{33}=+1, l_{1} & =-l_{2}\left[\arctan x_{2}+\frac{2 l_{2} x_{2}+1}{2 l_{2}\left(1+x_{2}^{2}\right)}\right], k=\frac{3+4 l_{2} x_{2}+x_{2}^{2}}{2\left(2 l_{2}-x_{2}\right)}, \alpha^{\prime}=k-x_{2} \\
x_{2} & <2 l_{2} \text { is the unique solution of : } \\
\arctan x_{2} & -\arctan \left[\frac{3+4 l_{2} x_{2}+x_{2}^{2}}{2\left(2 l_{2}-x_{2}\right)}\right]+\frac{3+2 l_{2} x_{2}+8 l_{2}^{2}}{6 l_{2}\left[1+\left(\frac{x_{2}+4 l_{2}}{3}\right)^{2}\right]}=0 .
\end{aligned}
$$

In particular, the position of the bolt is given by :

$$
\alpha^{\prime}=\frac{3\left(1+x_{2}^{2}\right)}{2\left(2 l_{2}-x_{2}\right)} \geq \frac{3}{2 l_{2}+\sqrt{4 l_{2}^{2}+1}}
$$

Notice that with $x_{2} \equiv-\left(4 l_{2}+3 x_{1}\right)$ the equations (16) become :

$$
\begin{aligned}
\frac{\pi}{2}+\arctan x_{1}+\frac{2 l_{2} x_{1}-1}{2 l_{2}\left(1+x_{1}^{2}\right)} & =0 \\
k=\frac{1+4 l_{2} x_{1}+3 x_{1}^{2}}{2\left(2 l_{2}+x_{1}\right)}, \quad l_{1} & =-l_{2}\left[\arctan k+\frac{k l_{2}-1}{l_{2}\left(1+k^{2}\right)}\right]
\end{aligned}
$$

with $x_{1}>-2 l_{2}$. One recognises equ.(15), but for the value of $l_{1}$. So, not too surprisingly, the metrics with a bolt at one end and a nut at the other end depend on a single transcendental equation (15) which characterises the presence of a bolt of twist 1.

$-\boldsymbol{\alpha}>\mathbf{0}$ : we have a bolt(p)-bolt(p) family of metrics (depending on a positive parameter $l_{2}$ ). The previous discussion enforces :

$$
n^{33}=+1, \mu=n^{33} l_{1}+l_{2}\left(\arctan k+\frac{k}{1+k^{2}}\right)>\frac{1}{1+k^{2}}, \delta=l_{1}-\frac{\pi}{2} l_{2}<0 .
$$

We have two bolt conditions (12) : one with $\mathrm{a}+p$ at $t=\alpha$, the other with $\mathrm{a}-p$ at $t=\alpha^{\prime}$. The vanishing of $\tilde{\Omega}^{2}(\alpha)$ and $\tilde{\Omega}^{2}\left(\alpha^{\prime}\right)$, will determine $p, l_{1}, \alpha, \alpha^{\prime}$ and $k$. As in the previous situation, we obtain :

$$
\begin{aligned}
& l_{1}=-l_{2}\left[\arctan x_{1}+\frac{2 l_{2} x_{1}-p}{2 l_{2}\left(1+x_{1}^{2}\right)}\right], k=x_{1}-\frac{(p-2)\left(1+x_{1}^{2}\right)}{2\left(p x_{1}+2 l_{2}\right)}, \alpha=k-x_{1}>0,(18) \\
& l_{1}=-l_{2}\left[\arctan x_{2}+\frac{2 l_{2} x_{2}+p}{2 l_{2}\left(1+x_{2}^{2}\right)}\right], k=x_{2}-\frac{(2+p)\left(1+x_{2}^{2}\right)}{2\left(p x_{2}-2 l_{2}\right)}, \alpha^{\prime}=k-x_{2}>\alpha>0 .
\end{aligned}
$$

\footnotetext{
${ }^{2} \mathrm{~A}$ useful result in the unicity discussion is the expression of the derivative with respect to $x_{2}$ of the left hand side of the equation in the third line of $(16)$ : it equals $\frac{19\left(2 l_{2}-x_{2}\right)}{l_{2}\left[9+\left(x_{2}+4 l_{2}\right)^{2}\right]^{2}}$.
} 
* The special value $p=2$ enforces $x_{1}=-l_{2}$ then $l_{1}=1+l_{2} \arctan \left(l_{2}\right)$. However, in that situation, the equation that determines $x_{2}$ has no solution.

* The difference of the two lines of equations (18) leads to the vanishing of two functions :

$$
\begin{aligned}
& f_{1}\left(x_{1}, x_{2}\right) \equiv-\arctan x_{1}+\arctan x_{2}-\frac{2 l_{2} x_{1}-p}{2 l_{2}\left(1+x_{1}^{2}\right)}+\frac{2 l_{2} x_{2}+p}{2 l_{2}\left(1+x_{2}^{2}\right)}=0, \\
& f_{2}\left(x_{1}, x_{2}\right) \equiv x_{1}-x_{2}-\frac{(p-2)\left(1+x_{1}^{2}\right)}{2\left(p x_{1}+2 l_{2}\right)}+\frac{(2+p)\left(1+x_{2}^{2}\right)}{2\left(p x_{2}-2 l_{2}\right)}=0
\end{aligned}
$$

For $p>2, \alpha^{\prime}>\alpha>0$ requires $x_{1}<-2 l_{2} / p$ and $x_{2}<2 l_{2} / p$. In this domain, the two partial derivatives of $f_{1}\left(x_{1}, x_{2}\right)$ are positive ; on the other hand

$$
f_{1}\left(x_{1}=x_{2}, x_{2}\right)=\frac{p}{l_{2}\left(1+x_{2}^{2}\right)}>0:
$$

as a consequence, $f_{1}\left(x_{1}>x_{2}, x_{2}\right)>f_{1}\left(x_{1}=x_{2}, x_{2}\right)$ cannot vanish. Then we have

Lemma 2 : Regular bolt-bolt Einstein-Weyl Bianchi IX metrics, non conformally Einstein, exist only with a twist $p=1$.

* For $p=1$, equation $f_{2}\left(x_{1}, x_{2}\right)=0$ gives either $x_{1}=-\frac{4 l_{2}+x_{2}}{3}$ or $x_{1}=\frac{2 l_{2} x_{2}+1}{2 l_{2}-x_{2}}$.

With regards to $f_{1}\left(x_{1}, x_{2}\right)$, when computing its total derivative with respect to $x_{2}$ with either solution for $x_{1}$, one readily finds that $f_{1}\left(\frac{2 l_{2} x_{2}+1}{2 l_{2}-x_{2}}, x_{2}\right)$ is the positive constant : $\left[1 /\left(2 l_{2}\right)-\arctan \left(1 /\left(2 l_{2}\right)\right)\right]$, which excludes that solution.

On the contrary, $f_{1}\left(-\frac{4 l_{2}+x_{2}}{3}, x_{2}\right)$ being monotonically increasing from $-\pi$ when $x_{2} \rightarrow-\infty$ to a positive value when $x_{2}=2 l_{2}$, one obtains one and only one solution.

To sum up, we have the following values for the parameters in the distance (3) :

$$
\begin{aligned}
& n^{33}=+1, l_{1}=-l_{2}\left[\arctan x_{2}+\frac{2 l_{2} x_{2}+1}{2 l_{2}\left(1+x_{2}^{2}\right)}\right], k=\frac{3+4 l_{2} x_{2}+x_{2}^{2}}{2\left(2 l_{2}-x_{2}\right)}, \\
& \quad \text { where } x_{2}<2 l_{2} \text { is the unique solution of }: f_{1}\left(-\frac{4 l_{2}+x_{2}}{3}, x_{2}\right)=0(.20)
\end{aligned}
$$

The positions of the bolts are given by

$$
\alpha=\frac{9+\left(4 l_{2}+x_{2}\right)^{2}}{6\left(2 l_{2}-x_{2}\right)} \geq \frac{1}{2 l_{2}+\sqrt{4 l_{2}^{2}+1}}, \alpha^{\prime}=\frac{3\left(1+x_{2}^{2}\right)}{2\left(2 l_{2}-x_{2}\right)} \geq \frac{3}{2 l_{2}+\sqrt{4 l_{2}^{2}+1}}, .
$$

- case $\left.\boldsymbol{c}^{-}\right)$: $\tilde{\boldsymbol{\Omega}}^{2}(\boldsymbol{t})$ decreases to a minimum value reached for $t=\beta, g(\beta)=0$, then increases and vanishes at $\alpha^{\prime}, \tilde{\Omega}^{2}\left(\alpha^{\prime}\right)=0$; the range of $t$ then goes to $+\infty$ which, requiring $\delta=0$, contradicts $\delta \mu<0$.

To summarize, in our Gauduchon's gauge we found four, and only four, families of regular Einstein-Weyl Bianchi $I X$ metrics; according to the classification of Gibbons and Hawking, they are complete and live on a compact manifold without boundary (see also in the concluding section the expression of the volume of the manifold) and have a constant positive conformal scalar curvature in agreement with the quoted theorem. 


\section{Bianchi $V I I_{0}$ regular metrics ?}

The metric write :

$$
\begin{aligned}
d s^{2} & =\frac{2}{\Gamma}\left[\frac{k}{\Omega^{2}(x)\left[1+x^{2}\right]^{2}}(d x)^{2}+\frac{k}{\left[1+x^{2}\right]}\left[\left(\sigma^{1}\right)^{2}+\left(\sigma^{2}\right)^{2}\right]+\frac{\Omega^{2}(x)}{k}\left(\sigma^{3}\right)^{2}\right], \\
\Omega^{2}(x) & =-2 k\left[x\left(l_{1}+l_{2} \arctan x\right)+l_{2}\right] .
\end{aligned}
$$

The positivity of the distance (21) enforces the two conditions

$$
k>0, \Omega^{2}(x)>0 .
$$

As

$$
\frac{d \Omega^{2}(x)}{d x}=-2 k h(x), \quad h(x)=l_{1}+l_{2}\left[\arctan x+\frac{x}{1+x^{2}}\right], \frac{d h(x)}{d x}=\frac{2 l_{2}}{\left[1+x^{2}\right]^{2}},
$$

here also the discussion separates into three subcases according to the behaviour of $\Omega^{2}(x)$ when $x$ varies. Let $\delta^{ \pm}=h( \pm \infty)=l_{1} \pm \frac{\pi}{2} l_{2}$ :

- a) $\delta^{ \pm} \leq 0: \Omega^{2}(x)$ increases with $x$.

The proper time $x$ belongs to the interval $\left[\alpha,+\infty\left[\right.\right.$, where $\Omega^{2}(\alpha)=0$.

- b) $\delta^{ \pm} \geq 0$ : $\Omega^{2}(x)$ decreases with $x$.

The proper time $x$ belongs to the interval ] $-\infty, \alpha]$. where $\Omega^{2}(\alpha)=0$.

- c) $\delta^{+} \delta^{-}<0: \Omega^{2}(x)$ has a single extremum when $x$ varies (obtained when $h(x)$ vanishes).

The extremum is a maximum if $l_{2}>0\left(\right.$ case $\left.c^{+}\right)$, a minimum if $l_{2}<0$ ( (ase $\left.c^{-}\right)$.

In the first case the maximum is readily seen to lead to $\Omega^{2}<0$; one remains with the sole case $c^{-}$, where the range of the proper time covers the whole $x$ axis $]-\infty,+\infty[$.

The behaviour of the distance at $\pm \infty$ is readily seen to be singular if $\delta^{ \pm} \neq 0$. Indeed,

$$
d s^{2} \sim \frac{2}{\Gamma}\left[\frac{(d x)^{2}}{\left(-2 \delta^{ \pm}\right) x^{5}}+\frac{k}{x^{2}}\left[\left(\sigma^{1}\right)^{2}+\left(\sigma^{2}\right)^{2}\right]-2 \delta^{ \pm} x\left(\sigma^{3}\right)^{2}\right], \quad x \rightarrow \pm \infty,
$$

and the change of variable $\rho=( \pm x)^{-3 / 2}$ leaves a non removable singularity at $\rho=0$.

To sum up, we have

Lemma 3 : in the Bianchi VII $I_{0}$ case, if the proper time interval extends to $+\infty$ (resp. $\left.-\infty\right)$, the metric can be regular only if $l_{1}+\frac{\pi}{2} l_{2}=0$ (resp. $\left.l_{1}-\frac{\pi}{2} l_{2}=0\right)$

Then the function $\Omega^{2}(x)$ simplifies to :

$$
\Omega^{2}(x)=-2 k l_{2}\left[1 \mp x\left(\frac{\pi}{2} \mp \arctan x\right)\right] \sim-2 k l_{2}\left[1-x \arctan \left(\frac{1}{x}\right)\right] \text { when } x \rightarrow \pm \infty .
$$

We now study successively the cases a), b) and $c^{-}$.

- case a) : $\boldsymbol{\Omega}^{\mathbf{2}}(\boldsymbol{x})$ monotonicaly increasing : only the case $\delta^{+}=0$ with $\delta^{-}<0$, i.e. $l_{2}>$ 0 has to be considered, then the function $\Omega^{2}(x)$ is always $<0$, which is excluded.

- case b) : $\Omega^{2}(x)$ monotonicaly decreasing : only the case $\delta^{-}=0$ with $\delta^{+}>$ 0 , i.e. $l_{2}>0$ has to be considered, but here again the function $\Omega^{2}(x)$ is always $<0$, which is excluded.

- case $\left.c^{-}\right)$: $\tilde{\Omega}^{2}(t)$ decreases to a minimum value then increases. One of the two ends of the $x$ axis remains singular.

Then there is no regular Einstein-Weyl metric in the Bianchi $V I I_{0}$ class. 


\section{Concluding remarks}

In this paper, we have presented an analysis of complete Einstein-Weyl structures $(g, \gamma)$ corresponding to Class A non-conformally Einstein Bianchi metrics in a Gauduchon's gauge. Thanks to our previous results, they are conformally Kähler. Let us recall that the extra $U(1)$ enlarging the Bianchi isometry group corresponds to the Killing vector dual to the Weyl form $\gamma$, and is responsible for the conformally Kähler property.

Firstly, we have found that there are no regular metric in the Bianchi $V I I_{0}$ and $V I I I$ subclasses ; this is not so surprising as in those cases, the underlying Lie group is non-compact : $U(1) \times E(3)$ (group of displacements in 3 dimensions) or $U(1) \times S U(1,1)$ respectively. Then, according to [3, 4] there cannot exist compact Einstein-Weyl solutions. On the contrary, Bianchi $I X$ Einstein-Weyl metrics have $U(1) \times S U(2)$ as isometry group which allows for a compact solution.

Secondly, we have shown how in each of the four families obtained, the completeness requirement determines all the parameters, but the homethetic one $\Gamma>0$, as soon as the (positive) conformal scalar curvature is fixed.

Let us now comment on their topological properties.

- Notice that in a Gauduchon's gauge, the Ricci tensor of the Levi Civita connection is positive definite as :

$$
R_{i j}=g_{i j} S^{D} / 4+\left(g_{i j}\|\gamma\|^{2}-\gamma_{i} \gamma_{j}\right) / 2,
$$

and the scalar curvature, related to the conformal scalar one through :

$$
R=S^{D}+\frac{3}{2}\|\gamma\|^{2} \Rightarrow R=4 \Gamma\left[l_{2}+\frac{3 \tilde{\Omega}^{2}(t)}{4 t}\right],
$$

is strictly positive on the manifold. As a consequence $\pi_{1}$ is finite and $b_{1}$ vanishes 9].

- The volume element writes :

$$
d \mathcal{V}=\frac{4 t}{\Gamma^{2}\left[1+(t-k)^{2}\right]^{2}} d t \sin \theta d \theta d \Phi d \Psi \Rightarrow \mathcal{V}=\left(\frac{4 \pi}{\Gamma}\right)^{2} \frac{V}{l_{2}}
$$

with $V=\frac{2 l_{2}+2 k}{1+k^{2}}$ (solution (14)), $V=\frac{2 l_{2}+k}{1+x^{2}}$ (solutions (15, 16)) and $V=\frac{2 l_{2}+k}{1+x^{2}}-\frac{2 l_{2}-k}{1+x^{\prime 2}}$ (solution (20)); this confirms the compact character of our metrics. But, if one goes to the Kähler gauge through the conformal transformation :

$$
\tilde{g}=\frac{\Gamma\left[1+(k-t)^{2}\right]}{2} g, \quad \tilde{\gamma}=\frac{\tilde{2}^{2}(t)}{2 t} \sigma^{3}+\frac{2(k-t) d t}{1+(k-t)^{2}}
$$

the volume element becomes :

$$
d \tilde{\mathcal{V}}=t d t \sin \theta d \theta d \phi d \Psi
$$

and only the two families with a finite range for the proper time stay compact. As explained in Section 2 (after equation (9)), for the first two families, an asymptotically euclidean boundary appears at $\infty$. 
- We have also computed the Euler number $\chi_{\mathrm{E}}$ and the signature $\tau$ for the compact metrics (14, 15, 16, 20) and found respectively : $\chi_{\mathrm{E}}=2,3,3$ or 4 , and $\tau=0,-1,+1$ or 0 , in agreement with [14] as a nut contributes 1 to the Euler number whereas a bolt contributes 2. For a compact four-dimensional manifold, $b_{0}=b_{4}=1$ and $b_{1}=b_{3}$; moreover, here $b_{1}$ vanishes, then one gets

$$
\chi_{\mathrm{E}}=2+b_{2}^{+}+b_{2}^{-}, \tau=b_{2}^{+}-b_{2}^{-},
$$

where $b_{2}^{ \pm}$is the number of self(anti-self)dual harmonic 2-forms on the manifold. The candidates harmonic 2-forms are :

$$
\begin{aligned}
& \omega^{+}=\left(d t \wedge \sigma^{3}+t \sigma^{1} \wedge \sigma^{2}\right)=\frac{\Gamma\left[1+(t-k)^{2}\right]}{2}\left[e_{0} \wedge e_{3}+e_{1} \wedge e_{2}\right], \\
& \omega^{-}=\left(d t \wedge \sigma^{3}-t \sigma^{1} \wedge \sigma^{2}\right) /\left(t^{2}\right)=\frac{\Gamma\left[1+(t-k)^{2}\right]}{2 t^{2}}\left[e_{0} \wedge e_{3}-e_{1} \wedge e_{2}\right] .
\end{aligned}
$$

Analysing the possible singularities of these 2-forms at the end points of the proper time interval, one easily sees that:

- our nut-nut family (14) has $b_{2}^{+}=b_{2}^{-}=0$ as the two 2-forms (25) are singular at $t=0$ or at $t \rightarrow+\infty$, then $\chi_{\mathrm{E}}=2$ and $\tau=0$;

- in the same way, the bolt-nut family (15) has $b_{2}^{+}=0$ and $b_{2}^{-}=1$, then $\chi_{\mathrm{E}}=3$ and $\tau=-1$ whereas the nut-bolt family (16) has $b_{2}^{-}=0$ and $b_{2}^{+}=1$, then $\chi_{\mathrm{E}}=3$ and $\tau$ $=+1$ (as remarked previously, these two families are similar but the orientation of the manifolds are opposite);

- finally, the bolt-bolt family (20) has $b_{2}^{+}=b_{2}^{-}=1$, then $\chi_{\mathrm{E}}=4$ and $\tau=0$.

Q.E.D.

Let us finally compare our results with thoses of Pedersen and Swann 90 and the systematic analysis of compact Einstein-Weyl structures with a large symmetry group done by Madsen in his PHD dissertation ([3]-especially Section 8), completed in [4](especially section 7) 0. The "time parameter" in these analyses is an angle $\varphi$.

Of course, our results are not new when compared to the global mathematical approach of [3, 4, but we think our language is more relevant for physicists and, moreover, our parametrisation being simpler, our discussion is able to correct some important mistakes.

- As the analysis of Gibbons and Hawking in the language of bolts and nuts applies only to orientable manifolds, it is not surprising that, contrary to [3, 4], we do not find as solutions, metrics on non-orientable manifolds such as $R P^{4}$ or $R P^{4} \# C P^{2}$.

- Our nut-nut family (14) corresponds to the solution where the manifold is $S^{4}$, the "time parameter" $\varphi \in\left[\varphi_{0}, \pi-\varphi_{0}\right]$ and special orbits at each end being a point (subsection 8-20 of [3], case 1 , page 426 of [4]).

- Our nut-bolt families (15,16) correspond to the solution where the manifold is $C P^{2}$ and special orbits are respectively a point and $C P^{1}$ (subsection 8-22 of [3]) : the (15) case corresponds to a "time parameter" $\varphi \in\left[\varphi_{0}, \Phi\right]$, special orbits being a point at $\varphi_{0}$ and

${ }^{3}$ When a first version of this work was completed, I was not aware of this publication, which explains some of the new comments. I thank the referees who drew my attention to this mathematical publication. 
$C P^{1}$ at $\Phi$ (in the notations of Madsen) ; the (16) case corresponds to a "time parameter" $\varphi \in\left[\Phi, \pi-\varphi_{0}\right]$, special orbits being a point at $\pi-\varphi_{0}$ and $C P^{1}$ at $\Phi$. In [4], an isolated solution is also given (case $3 \mathrm{a}$, page 427 ) ; however, this is wrong : an easy calculation checks that, contrary to their assertion, it does belong to the previous one-parameter family.

- Our bolt-bolt family (20) corresponds to the solution where the "time parameter" $\varphi \in$ $\left[\Phi_{1}, \Phi_{2}\right]$, and special orbits are both $C P^{1}$ (subsection 8-24 of [3]), but we have been able to prove that no bolt(p)-bolt(p) exists with $p \geq 2$ (Lemma 2), a result which was only conjectured by Madsen. Moreover, thanks to our parametrisation that disentangles the parameters $k, x_{1}$ and $x_{2}$ into a single transcendental equation for only one unknown parameter, we also proved that the relation $\Phi_{1}+\Phi_{2}=\pi$, (c.f. $3 x_{1}+x_{2}=-4 l_{2}$ ), also conjectured in Madsen's thesis dissertation, is indeed the sole solution. In [佃, it is also proven, with some efforts, that the conjectured relation $\Phi_{1}+\Phi_{2}=\pi$ holds. The authors claimed too that, apart from $p=1$, solutions exist for any $p \geq 4$; however in their proof (pages 429-430), they forget about a constraint on their parameter $\chi$ which has to be at least equal to their parameter $D$; therefore, $\tan (\chi / 2)$, which they found

must be greater than $\frac{2}{p} \tan (D / 2)$, should also be greater than $\tan (D / 2)$ which is a more restrictive condition as soon as $p>2$. When this condition is added, one finds that no bolt(p)-bolt(p) exists with $p \geq 2$.

As a final remark, note that the manifold is the Hirzebruch surface $C P^{2} \# \overline{C P^{2}}$, as in the family firstly exhibited by Pedersen and Swann [9]. Several other metrics are known to live on this manifold (Page's Einstein metric [15], Calabi's extremal Kähler metric [16], Chave and Valent's quasi-Einstein Kähler metric [17]).

Our results may be summarized in the

Theorem : In a Gauduchon's gauge, non-exact Einstein-Weyl structures $(g, \gamma)$ corresponding to class $A$ (non-)diagonal Bianchi metrics on an orientable manifold are regular only when $[\mathrm{g}]$ belongs to the Bianchi IX subclass:

i) the metrics are conformally Kähler,

ii) they have an arbitrary constant positive conformal scalar curvature,

iii) they occur in four one-parameter families characterised by their end-points being respectively nut-nut, nut-bolt(1), bolt(1)-nut and bolt(1)-bolt(1),

iv) they live on the compact manifolds $S^{4}, C P^{2}$ (with two possible orientations) and $C P^{2} \# \overline{C P^{2}}$ respectively .

\section{References}

[1] H. Pedersen and A. Swann, Proc. Lond. Math. Soc. 66 (1993) 381.

[2] D.M.J. Calderbank and H. Pedersen, "Einstein-Weyl geometry", Edinburgh Preprint MS98-010, to appear in "Essays on Einstein manifolds", International Press.

[3] A. Madsen, "Compact Einstein-Weyl manifolds with large symmetry group", PhD. Thesis, Odense University, 1995.

[4] A.B. Madsen, H. Pedersen, Y.S. Poon and A.F. Swann, Duke Math. J. 88 (1997) 407. 
[5] R. Gauduchon, Math. Ann. 267 (1984) 495.

[6] K. P. Tod, J. London Math.Soc. 245(1992) 341.

[7] H. Pedersen and K. P. Tod, Adv. Math. 1(1993) 74.

[8] P. Gauduchon, "Structures de Weyl-Einstein, Espaces de twisteurs et Variétés de type $S^{1} \times S^{3} "$, unpublished preprint.

[9] H. Pedersen and A. Swann, J. Reine Angew. Math. 441 (1993) 99.

[10] G. Bonneau, Class. Quantum Grav. 15 (1998) 2415.

[11] A. S. Dancer and Ian A. B. Straham, Cohomogeneity-One Kähler metrics in "Twistor theory", S. Huggett ed., Marcel Dekker Inc., New York, 1995, p.9.

[12] K. P. Tod, Cohomogeneity-One metrics with Self-Dual Weyl tensor in "Twistor theory", S. Huggett ed., Marcel Dekker Inc., New York, 1995, p.171.

[13] G. W. Gibbons and S. W. Hawking, Commun. Math. Phys. 66 (1979) 291 ; G. W. Gibbons and C. N. Pope, Commun. Math. Phys. 66 (1979) 267.

[14] T. Eguchi, P. B. Gilkey and A.J. Hanson, Phys. Reports 66 (1980) 213.

[15] D. Page, Phys. Lett. B79 (1978)235.

[16] E. Calabi, Extremal Kähler metrics in "Seminars on differential geometry", S.T. Yau ed., Annals of Math. Stud. (Princeton Univ; press, Princeton, 1982) p. 259.

[17] T. Chave and G. Valent, Nucl. Phys. B478 (1996) 758. 